\title{
Balanço hídrico da cultura da mamona sob condições de sequeiro
}

\author{
Cícero de Souza ${ }^{1}$, Alberício P. de Andrade ${ }^{2}$, José R. de S. Lima ${ }^{3}$, \\ Antonio C. D. Antonino ${ }^{4}$, Eduardo S. de Souza ${ }^{5}$ \& Ivandro de F. da Silva ${ }^{1}$
}

\begin{abstract}
RESUMO
O estudo dos componentes do balanço de água no solo é essencial para se compreender sua dinâmica, fornecendo subsídios para o manejo agrícola e otimização do uso de água. O presente trabalho objetivou determinar os componentes do balanço de água em solo cultivado com mamona sob condições de sequeiro. Para tal foram instalados, numa área de 4 ha da Fazenda Experimental Chã do Jardim, do Centro

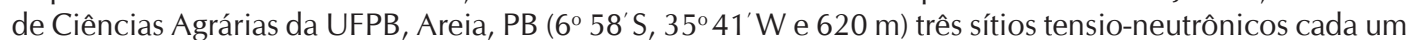
contendo um tubo de acesso para a sonda de nêutrons até a profundidade de 1,60 $\mathrm{m}$ e oito tensiômetros nas profundidades de 0,$10 ; 0,20 ; 0,30 ; 0,40 ; 0,60 ; 0,80 ; 1,20$ e 1,60 m, além de um pluviógrafo para medir a precipitação. Com vista à determinação da condutividade hidráulica saturada do solo, foram realizados ensaios de infiltração na superfície e nas profundidades de 0,20; 0,40;0,60 e 0,80 m. A evapotranspiração da mamona seguiu as variações da precipitação e teve valor médio de $2,43 \mathrm{~mm} \mathrm{~d}^{-1}$, perfazendo o total de 342,52 mm. As variações do armazenamento de água no solo seguiram as variações da precipitação pluvial e o fluxo de água na base do solo (ascensão capilar ou drenagem) tiveram valores muito pequenos.
\end{abstract}

Palavras-chave: Ricinus communis L., evapotranspiração, drenagem, umidade do solo

\section{Water balance of castor bean under rainfed conditions}

\begin{abstract}
The study of water balance components is essential to understand the dynamics of soil water, providing subsidies to agricultural management and optimization of water use. The objective of this study was to determine the water balance components in a soil cultivated with castor bean under rainfed conditions. An experiment was installed in a 4 ha area in the Centro de Ciências Agrárias, UFPB, in the municipality of Areia, Paraiba state $\left(6^{\circ} 58^{\prime} \mathrm{S}, 35^{\circ} 41^{\prime} \mathrm{W}\right.$ e $\left.620 \mathrm{~m}\right)$, a rain gauge and three plots contained an access tube for neutron probe measurements, up to the depth of $1.60 \mathrm{~m}$, and a set of eight tensiometers at depths of $0.10,0.20,0.30,0.40,0.60,0.80,1.20$ and $1.60 \mathrm{~m}$. Infiltration tests were performed on soil surface and $0.20,0.40,0.60$ and $0.80 \mathrm{~m}$ depth for the determination of the saturated hydraulic conductivity of soil. It was verified that the evapotranspiration of castor bean followed the variations of rainfall with the mean and total values of $2.43 \mathrm{~mm} \mathrm{~d}^{-1}$ and $342.5 \mathrm{~mm}$, respectively. The variation of soil moisture storage change followed the variations in rainfall and the flux of water crossing the last soil layer (capillarity rise or drainage) had very small values.
\end{abstract}

Key words: Ricinus communis L., evapotranspiration, drainage, soil water content

CCA/UFPB, Campus II, CEP 58397-000, Areia, PB. Telefone (83) 3362-2300. E-mail: cicerosolos@yahoo.com.br; ivandro@cca.ufpb.br ${ }^{2}$ CCA/UFPB, UAG/UFRPE, Rua Newton Estilac Leal, 1189, Alto Branco, CEP 58401-750, Campina Grande, PB. Fone: (83) 3343-1050. E-mail: albericio@pq.cnpq.br

${ }^{3}$ UAG/UFRPE, Av. Bom Pastor S/N, Boa Vista, CEP 55292-270, Garanhuns, PE. Fone: (87) 3764-5500. E-mail: romualdo@uag.ufrpe.br

${ }^{4}$ DEN/UFPE, Av. Prof. Luiz Freire, 1000, Cidade Universitária, CEP 50740-540, Recife, PE. Fone: (81) 2126-7973

${ }_{5}^{5}$ UAST/UFRPE, Fazenda Saco, s/n, C.P. 63, CEP 56900-000, Serra Talhada, PE. Fone: (87) 3431-1927. E-mail: eduardosouza@uast.ufrpe.br 


\section{INTRODUÇÃO}

Através do Probiodiesel o Governo Federal tem incentivado, nos últimos anos, o cultivo de plantas oleaginosas de acordo com o potencial de cada região, visando à produção do biodiesel. No Nordeste brasileiro e devido às condições edafoclimáticas, a cultura escolhida para a produção de biodiesel foi a mamona pois, além de sua adaptabilidade a essas condições, apresenta elevada potencialidade para gerar empregos e fixar o homem no campo, o que diminui o êxodo rural (Oliveira et al., 2009).

A faixa ideal de precipitação para a produção da mamona varia entre 750 e $1500 \mathrm{~mm}$, com um mínimo de 600 a 750 mm durante todo o ciclo da cultura ajustando-se o plantio, de forma que a planta receba de 400 a $500 \mathrm{~mm}$ até o início da floração (Távora, 1982). Em regiões que apresentam totais de precipitação inferiores a $500 \mathrm{~mm}$ no período chuvoso, a mamoneira perde grande parte da sua produção econômica, acentuando-se os riscos de perda total de safras e/ou a obtenção de rendimento muito baixo (Barros Júnior et al., 2008). No entanto, apenas os dados de precipitação pluvial não são suficientes para se prever a quantidade de água disponível no solo para as culturas uma vez que esses informam tão somente a entrada de água no solo. Faz-se necessário conhecer também as perdas de água do solo, quer sejam as relacionadas à evapotranspiração ou aquelas relacionadas com a drenagem e com o escoamento superficial, evidenciando a necessidade de se realizar o balanço hídrico no solo.

De acordo com Brito et al. (2009) o método do balanço hídrico mostra que durante dado período a soma algébrica de todas as entradas (consideradas positivas) e saídas (negativas) de água em um volume de controle de solo submetido às peculiaridades dos fenômenos meteorológicos, dos atributos do solo e das características genéticas da espécie ou mesmo da variedade vegetal, resulta no saldo ou no balanço de água no solo, no período. No campo considera-se este volume delimitado por duas superfícies paralelas: pela interface solo-atmosfera (limite superior do volume ou simplesmente superfície do solo) por uma superfície horizontal localizada na profundidade do sistema radicular do cultivo (limite inferior do volume).

A importância do balanço hídrico no solo como ferramenta para avaliar a intensidade das saídas e as entradas de água no solo e, por conseguinte, para definição dos períodos mais prováveis de déficit hídrico para a cultura, está relacionada não só ao conhecimento dos fatores que o compõem (evapotranspiração, precipitação, drenagem, ascensão capilar, escoamento superficial) mas também ao conhecimento das características da planta, principalmente da sua fenologia, que representa o ponto de partida para a interpretação coerente dos resultados do balanço (Cintra et al., 2000).

O método do balanço hídrico no solo vem sendo muito utilizado para se estimar a evapotranspiração e a eficiência no uso de água de várias culturas (Cruz et al., 2005b; Lima et al., 2006b; Moroke et al., 2011; Ward et al., 2012) por apresentar boa concordância quando comparado com os lisímetros, além de ser mais barato (Lima et al., 2006a ).

Deste modo, o presente trabalho teve como objetivo avaliar os componentes do balanço hídrico (precipitação pluvial, variação do armazenamento de água, ascensão capilar, drenagem interna e evapotranspiração) em solo cultivado com a cultura da mamona, sob condições de sequeiro, na região do Brejo Paraibano.

\section{Material E MÉtodos}

As medidas para realização do balanço de água no solo foram efetuadas numa área de 4 ha do Centro de Ciências Agrárias da Universidade Federal da Paraíba, localizada no município de Areia, PB (6 $6^{\circ} 58^{\prime} 12^{\prime}$ " S e $35^{\circ} 42^{\prime} 15^{\prime}$ " W e $620 \mathrm{~m}$ de altitude). O clima na região, pela classificação de Köppen, é do tipo As' (quente e úmido). De acordo com os dados da Estação Meteorológica do CCA da UFPB (média de 30 anos) a temperatura média anual é de $24,5{ }^{\circ} \mathrm{C}$, a umidade relativa média anual é de $85 \%$ e a precipitação pluvial anual é de 1.400 mm. O quadrimestre mais chuvoso é constituído dos meses de abril, maio, junho e julho, e representa $62 \%$ do total médio anual (Oliveira et al., 2009).

O solo da área, classificado como Latossolo Amarelo (EMBRAPA, 2006) apresentou as seguintes características químicas determinadas utilizando-se as metodologias recomendadas pela EMBRAPA (1997): $\mathrm{pH}$ em água 5,2; $\mathrm{P}$ e $\mathrm{K}$ extraídos pelo método de Mehlich, 0,75 e $30 \mathrm{mg} \mathrm{dm}^{-3}$, respectivamente; matéria orgânica, $48 \mathrm{~g} \mathrm{dm}^{-3}$ e CTC, 10,90 $\mathrm{cmol}_{\mathrm{c}} \mathrm{dm}^{-3}$. Também foram realizadas análises físicas do solo, constando de granulometria e densidade do solo, em amostras coletadas a cada $0,20 \mathrm{~m}$, até a profundidade de $0,80 \mathrm{~m}$ (Tabela 1). A fração areia foi obtida por peneiramento e as frações de argila e de silte foram determinadas por sedimentação, após dispersão com hexametafosfato de sódio, utilizando-se o método do densímetro (EMBRAPA, 1997). A densidade do solo foi determinada utilizando-se o método do anel volumétrico (EMBRAPA, 1997).

Tabela 1. Análise granulométrica, classificação textural e densidade do solo $(\rho)$ da área experimental

\begin{tabular}{|c|c|c|c|c|c|}
\hline \multirow{2}{*}{$\begin{array}{l}\text { Profundidade } \\
\text { (m) }\end{array}$} & \multicolumn{3}{|c|}{ Granulometria $\left(\mathrm{g} \mathrm{kg}^{-1}\right)$} & \multirow{2}{*}{$\begin{array}{c}\text { Classificação } \\
\text { textural* }\end{array}$} & \multirow{2}{*}{$\begin{array}{c}* * p \\
\left(\mathrm{~kg} \mathrm{dm}^{-3}\right)\end{array}$} \\
\hline & Areia & Silte & Argila & & \\
\hline $0-0,20$ & 628,9 & 140,7 & 230,4 & FAA & 1,28 \\
\hline $0,20-0,40$ & 558,2 & 140,8 & 301,0 & FAA & 1,33 \\
\hline $0,40-0,60$ & 470,2 & 117,3 & 412,5 & $A A$ & 1,37 \\
\hline $0,60-0,80$ & 488,7 & 82,0 & 429,3 & $A A$ & 1,31 \\
\hline
\end{tabular}

* FAA - Franco argilo arenosa, AA - Argilo arenosa

Ao final do experimento fez-se uma trincheira entre as plantas de mamona e se observou que a profundidade efetiva do sistema radicular se encontrava aos 0,60 m. Deste modo, o balanço de água foi efetuado considerando-se o perfil de solo de 0-0,60 m. Ressalta-se que as raízes mais profundas, as de sustentação, podem alcançar profundidades superiores a $1,5 \mathrm{~m}$ (Azevedo \& Lima, 2001).

O período de estudo do balanço de água no solo foi de 06/07/2005 a 24/11/2005, totalizando 141 dias e foi dividido em 14 subperíodos, sendo 7 com 10 dias, 2 com 11 dias, $1 \mathrm{com}$ 13 dias, 1 com 12 dias, 1 com 9 dias, 1 com 8 dias e 1 com 7 dias. A não uniformidade dos subperíodos em número de dias foi devido às leituras de umidade volumétrica e de potencial total de água do solo apresentarem algumas lacunas. 
Em referência à determinação do balanço de água no solo foram instalados três sítios tensioneutrônicos, cada qual contendo um tubo de acesso, em alumínio, para a sonda de nêutrons e oito tensiômetros (de manômetro de mercúrio) instalados nas profundidades de 0,$10 ; 0,20 ; 0,30 ; 0,40 ; 0,60$; 0,$80 ; 1,20$ e 1,60 m. As medidas neutrônicas foram realizadas diariamente (às $8 \mathrm{~h}$ ) a cada $0,10 \mathrm{~m}$, em três repetições, até a profundidade de $0,80 \mathrm{~m}$; após esta profundidade as leituras eram feitas de 0,20 em 0,20 m até a profundidade de 1,60 m, com uma sonda Troxler série 4300, tendo a mesma uma fonte radioativa de $\mathrm{Am}-\mathrm{Be}$ de atividade de $10 \mathrm{~m} \mathrm{Ci}(0,37 \mathrm{GBq})$ e tempo de contagem de $60 \mathrm{~s}$. Durante as medidas utilizou-se um disco refletor com $0,20 \mathrm{~m}$ de diâmetro para minimizar a fuga de nêutrons pela superfície do solo. Além disto, foram seguidos todos os procedimentos de proteção radiológica, de acordo com Oresegun (2000). As leituras nos tensiômetros também foram realizadas diariamente no mesmo horário das leituras neutrônicas.

Obteve-se a curva de calibração da sonda de nêutrons a partir da relação entre a umidade volumétrica e a contagem normalizada da sonda para as camadas de 0,10-0,60 m (Figura 1).

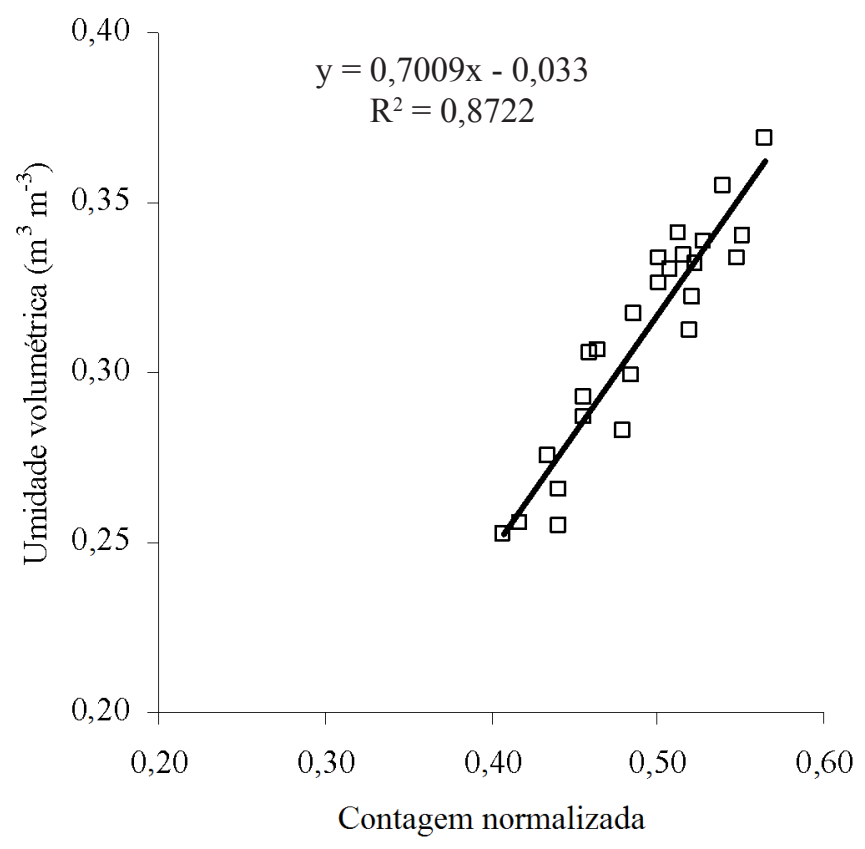

Figura 1. Calibração da sonda de nêutrons para a camada de 0,10-0,60 m de um Latossolo Amarelo em Areia, PB

As umidades volumétricas foram obtidas a partir de determinações gravimétricas de três amostras, coletadas com amostrador de Ulhand, com cilindros de $0,07 \mathrm{~m}$ de diâmetro e $0,083 \mathrm{~m}$ de altura, próximo ao tubo de acesso de cada sítiotensio-neutrônico, logo após serem realizadas as medidas com a sonda de nêutrons; essas amostras foram pesadas e secadas em estufa a $105^{\circ} \mathrm{C}$, até peso constante.

O plantio da mamona foi realizado manualmente, nos dias 31/05 e 01/06/2005, em área preparada com tração mecânica (aração e gradagem), no espaçamento de 2,0 x 1,0 m (Azevedo \& Lima, 2001), sendo colocadas três sementes por cova da cultivar BRS 149 Nordestina, que tem porte médio, sementes grandes (média de $68 \mathrm{~g}$ por 100 sementes), ciclo médio e frutos semi-indeiscentes (Beltrão et al., 2003). Foram realizadas duas capinas durante o ciclo vegetativo da cultura, aos 45 e 75 dias após o plantio.

Uma adubação foi realizada constando de $300 \mathrm{~kg} \mathrm{ha}^{-1} \mathrm{de}$ sulfato de amônia, $178 \mathrm{~kg} \mathrm{ha}^{-1}$ de superfosfato simples e 100 $\mathrm{kg} \mathrm{ha}^{-1}$ de cloreto de potássio, de acordo com os resultados de análise de fertilidade do solo. A adubação de cobertura foi realizada nos dias 09/08 e 10/08/2005, efetuada em sulcos distantes $0,10 \mathrm{~m}$ da linha das plantas.

O balanço de água em determinado volume de solo e pela lei de conservação das massas em certo período de tempo (Lima et al., 2006b) é descrito pela Eq. 1:

$$
\Delta \mathrm{A}=\mathrm{P}+\mathrm{I}+\mathrm{AC}-\mathrm{D} \pm \mathrm{R}-\mathrm{ET}
$$

em que:

$\Delta \mathrm{A}$ - variação no armazenamento de água no perfil de solo, $\mathrm{mm}$

P - precipitação pluvial, mm

I - irrigação, mm

AC - ascensão capilar, mm

D - drenagem interna, $\mathrm{mm}$

$\mathrm{R}$ - escoamento superficial, $\mathrm{mm}$

ET - evapotranspiração real, $\mathrm{mm}$

A precipitação pluvial (P) foi monitorada por meio de um pluviógrafo automatizado (TE 525WS-L, Texas Eletronics) instalado no centro da área experimental. O termo irrigação (I) foi nulo, uma vez que o trabalho foi realizado em condições de sequeiro. $\mathrm{O}$ escoamento superficial de água (R) não foi considerado em razão do solo apresentar topografia plana, bem estruturado, profundo, naturalmente bem drenado, além do plantio em nível da mamoneira.

$\mathrm{O}$ armazenamento acumulado de água no solo $\left(\mathrm{A}_{\mathrm{L}}\right)$ foi calculado pela regra do trapézio considerando-se que as medidas foram realizadas em intervalos igualmente espaçados, desde a superfície $(\mathrm{z}=0)$ até a profundidade de interesse $(\mathrm{z}=$ L) através da Eq. 2:

$$
A_{L}=\int_{0}^{L} \theta(z) d z=\left[0,50 \theta(z)+\sum_{i=1}^{n-1} \theta\left(z_{i}\right)+0,50 \theta\left(z_{n}\right)\right] \Delta z
$$

em que:

$\theta \quad$ - umidade volumétrica do solo, $\mathrm{m}^{3} \mathrm{~m}^{-3}$

A variação no armazenamento de água no perfil de solo $(\Delta \mathrm{A})$ foi determinada pela diferença dos valores de umidade volumétrica obtidos do perfil nos tempos inicial e final de cada período considerado:

$$
\Delta \mathrm{A}=\left[\theta_{(\mathrm{f})}-\theta_{(\mathrm{i})}\right] \mathrm{L}=\mathrm{A}_{\mathrm{f}}-\mathrm{A}_{\mathrm{i}}
$$

em que:

$\mathrm{A}_{\mathrm{i}}$ e $\mathrm{A}_{\mathrm{f}}$ os armazenamentos $(\mathrm{mm})$ acumulados de água inicial e final, respectivamente. 
As perdas e/ou os ganhos de água através da base do perfil de solo estudado, ou seja, drenagem interna (D) ou ascensão capilar (AC) foram estimadas com base na equação de DarcyBuckingham:

$$
\mathrm{q}=-\mathrm{K}(\theta) \nabla \psi_{\mathrm{t}}
$$

em que:

q - densidade de fluxo da água no solo, $\mathrm{mm} \mathrm{d}^{-1}$ (valor positivo indica $\mathrm{AC}$ e negativo, $\mathrm{D})$

$\mathrm{K}(\theta)$ - condutividade hidráulica do solo, $\mathrm{mm} \mathrm{d}^{-1}$

$\nabla \psi_{\mathrm{t}}$ - gradiente de potencial total na direção vertical, $\mathrm{cm}$ $\mathrm{cm}^{-1}$

O gradiente de potencial total foi calculado a partir dos potenciais totais obtidos nas profundidades de 0,40 e $0,80 \mathrm{~m}$. O potencial total da água no solo $\left(\psi_{t}\right)$ foi obtido pela Eq. 5:

$$
\psi_{\mathrm{t}}=\psi_{\mathrm{m}}+\psi_{\mathrm{g}}
$$

em que:

$\psi_{\mathrm{g}} \quad$ - potencial gravitacional ( $\mathrm{cm}$ água), igual à profundidade

$\psi_{\mathrm{m}} \quad$ - potencial matricial (cm água)

O potencial matricial $\left(\psi_{\mathrm{m}}\right)$ foi obtido das leituras nos tensiômetros por meio da Eq. 6:

$$
\psi_{\mathrm{m}}=-12,6 \mathrm{~h}+\mathrm{h} 1+\mathrm{h} 2
$$

em que:

h - leitura em $\mathrm{cm}$ de mercúrio

h1 - altura do manômetro (cm água) em relação a superfície do solo

h2 - profundidade (cm água) da cápsula porosa em relação à superfície do solo

A curva de retenção foi obtida a partir dos dados de potencial matricial e de umidade do solo obtidos em campo, por meio dos tensiômetros e da sonda de nêutrons, respectivamente. A curva de retenção foi complementada para valores acima de 0,1 MPa em laboratório usando-se Extrator de Richards, de acordo com metodologia da EMBRAPA (1997). Os dados das curvas de retenção da água no solo foram ajustados por intermédio da equação proposta por Genuchten (1980):

$$
\theta(\mathrm{h})=\theta_{\mathrm{r}}+\left(\theta_{\mathrm{s}}-\theta_{\mathrm{r}}\right) \times\left[1+\left(\alpha \psi_{\mathrm{m}}\right)^{\mathrm{n}}\right]^{\mathrm{m}}
$$

com a hipótese de Burdine (1953):

$$
\mathrm{m}=1-\frac{2}{\mathrm{n}}
$$

em que:

$$
\begin{array}{ll}
\theta_{\mathrm{s}} & \text { - umidade volumétrica do solo na saturação, } \mathrm{m}^{3} \mathrm{~m}^{-3} \\
\theta_{\mathrm{r}} & - \text { umidade volumétrica residual, } \mathrm{m}^{3} \mathrm{~m}^{-3}
\end{array}
$$

$\alpha \quad$ - representa o inverso da pressão de borbulhamento $\left(\mathrm{cm}^{-1}\right)$, a partir da qual a água começa a ser drenada do solo previamente saturado

n e m - parâmetros de ajuste da equação

O parâmetro $\theta_{\mathrm{s}}$ foi obtido por pesagem direta das amostras após o processo de saturação. Os parâmetros $\alpha$ e $n$ foram obtidos por meio da rotina Solver da planilha eletrônica Excel. Os valores de $\theta$ foram considerados iguais a 0,0 (zero) de acordo com Wessolek et al. (1994).

A condutividade hidráulica não saturada $K(\theta)$ foi obtida por intermédio da equação de Genuchten (1980) utilizando-se a hipótese de Burdine (1953):

$$
\mathrm{K}(\theta)=\mathrm{K}_{\mathrm{s}} \mathrm{S}_{\mathrm{e}}\left[1-\left(1-\mathrm{S}_{\mathrm{e}}^{1 / \mathrm{m}}\right)^{\mathrm{m}}\right]
$$

em que:

$\mathrm{K}_{\mathrm{s}} \quad$ - condutividade hidráulica saturada do solo, $\mathrm{mm} \mathrm{d}^{-1}$

$\mathrm{S}_{\mathrm{e}} \quad$ - umidade efetiva, adimensional

A umidade efetiva $\left(\mathrm{S}_{\mathrm{e}}\right)$ foi obtida pela relação:

$$
\mathrm{S}_{\mathrm{e}}=\frac{\theta-\theta_{\mathrm{r}}}{\theta_{\mathrm{s}}-\theta_{\mathrm{r}}}
$$

Para estimar $\mathrm{K}_{\mathrm{s}}$ foram realizados ensaios de infiltração com infiltrômetro de anel simples, com $0,15 \mathrm{~m}$ de diâmetro na superfície e nas profundidades de 0,20;0,40;0,60 e 0,80 m, utilizando-se o método proposto por Haverkamp et al. (1994). O ensaio de infiltração consiste em se anotar o tempo que volumes constantes de água levam para infiltrar no solo, esses volumes de água podem variar de 70 a $250 \mathrm{~mL}$, dependendo da taxa de infiltração, a fim de evitar uma carga hidráulica e consequente fluxo forçado no solo. A infiltração acumulada é obtida calculando-se a razão entre o volume acumulado e a área do infiltrômetro de anel $\left(\mathrm{A}=0,018 \mathrm{~m}^{2}\right)$.

Haverkamp et al. (1994) propuseram a seguinte equação para a infiltração acumulada para tempos longos:

$$
\mathrm{I}_{3 \mathrm{D}}=\mathrm{K}_{\mathrm{s}}+\frac{\gamma \mathrm{S}_{\mathrm{o}}^{2}}{\mathrm{r} \Delta} \mathrm{t}+\frac{\mathrm{S}_{\mathrm{o}}^{2}}{2\left(\mathrm{~K}_{\mathrm{s}}\right)(1+\beta)} \ln \frac{1}{\beta}
$$

em que:

$\mathrm{r} \quad$ - raio do disco, $\mathrm{m}$

$\mathrm{S}_{\mathrm{o}} \quad$ - sorvidade, $\mathrm{mm} \mathrm{d}^{-1}$

$\Delta \theta$ - variação de umidade volumétrica, $\mathrm{m}^{3} \mathrm{~m}^{-3}$

$\beta$ - constante no intervalo $(0<\beta<1)$ cujo valor é igual a 0,6 (Haverkamp et al., 1994)

$\gamma \quad$ - constante teórica, cujo valor é igual a 0,75

A condutividade hidráulica saturada $\left(\mathrm{K}_{\mathrm{s}}\right)$ do solo foi obtida por intermédio do ajuste da Eq. 11 aos dados da infiltração acumulada em função do tempo por intermédio da sub-rotina DBCONF da IMSL (IMSL, 1989). 
Os valores dos parâmetros da curva de retenção obtidos por meio do ajuste da Eq. 7 e os valores de $\mathrm{K}_{\mathrm{s}}$ estão apresentados na Tabela 2 .

Tabela 2. Valores dos parâmetros da curva de retenção da água no solo, $\theta(\mathrm{h})$, equação de Genuchten (1980) e de condutividade hidráulica saturada $\left(\mathrm{K}_{\mathrm{s}}\right)$ para as profundidades de 0,$20 ; 0,40 ; 0,60$ e 0,80 m

\begin{tabular}{ccccccc}
\hline $\begin{array}{c}\text { Prof. } \\
\mathbf{m}\end{array}$ & $\begin{array}{c}\theta \mathbf{S} \\
\mathbf{m}^{3} \mathbf{~ m}^{\mathbf{3}}\end{array}$ & $\begin{array}{c}\boldsymbol{\alpha} \\
\mathbf{c m}^{-1}\end{array}$ & $\mathbf{n}$ & $\mathbf{m}$ & $\mathbf{R}^{2}$ & $\begin{array}{c}\mathbf{K}_{\mathbf{s}} \\
\mathbf{m m ~ d}^{-1}\end{array}$ \\
0,20 & 0,4260 & 3,3108 & 2,0759 & 0,0366 & 0,9298 & $2.848,85$ \\
0,40 & 0,4653 & 0,8238 & 2,0932 & 0,0445 & 0,9405 & 468,10 \\
0,60 & 0,4588 & 0,2282 & 2,1150 & 0,0544 & 0,8895 & 527,89 \\
0,80 & 0,4574 & 1,0914 & 2,0778 & 0,0374 & 0,8901 & 481,24 \\
\hline
\end{tabular}

A ETo foi obtida pelo método de Penman-Monteith, de acordo com Allen et al. (1998). Os dados necessários (temperatura e umidade relativa do ar, velocidade do vento, saldo de radiação e fluxo de calor no solo) foram obtidos de uma estação meteorológica automática instalada no centro da área. Maiores detalhes quanto a esta estação podem ser obtidos em Oliveira et al. (2009).

Apesar do fluxo de calor no solo ser considerado nulo no período de um dia (Allen et al., 1998) diversos autores (Lima et al., 2011; Krishnan et al., 2012) têm encontrado que o fluxo de calor no solo pode ser de 10 a $30 \%$ do saldo de radiação no período diário. Assim, o fluxo de calor no solo $(\mathrm{G})$ foi obtido por meio da Eq. 12 (Lima et al., 2011):

$$
\mathrm{G}=\mathrm{G}_{\mathrm{p}}+\frac{\Delta \mathrm{T}_{\mathrm{s}} \mathrm{CD}}{\mathrm{t}}
$$

em que:

$\mathrm{G}_{\mathrm{p}} \quad$ - fluxo de calor medido pelo fluxímetro $\left(\mathrm{W} \mathrm{m}^{-2}\right)$

$\Delta \mathrm{T}_{\mathrm{S}}$ - variação na temperatura média do solo $\left({ }^{\circ} \mathrm{C}\right)$ durante o período de medição

C - capacidade térmica volumétrica do solo $\left(\mathrm{MJ} \mathrm{m}^{-3}{ }^{\circ} \mathrm{C}^{-1}\right)$

D - profundidade do fluxímetro $(\mathrm{m})$

t $\quad$ - duração do período de medição (s)

O fluxímetro (modelo HFT3 da Campbell Scientific Inc.) foi instalado entre plantas de mamona na profundidade de 0,05 $\mathrm{m}$. No mesmo local também foram instalados dois sensores para medir a temperatura do solo nas profundidades de 0,02 e 0,08 m e um sensor tipo TDR (modelo CS615 da Campbell Scientific Inc.) para medir a umidade volumétrica do solo na profundidade de $0,05 \mathrm{~m}$.

$\mathrm{O}$ valor de $\mathrm{C}$ foi estimado somando-se as capacidades caloríficas dos vários constituintes do solo, ponderados de acordo com suas frações de volume, com base em Vries (1966):

$$
\mathrm{C}=1,92 \mathrm{f}_{\mathrm{m}}+2,51 \mathrm{f}_{0}+4,18 \theta
$$

em que:

$f_{m} \quad$ - fração de volume dos minerais, 0,47

$\mathrm{f}_{\mathrm{o}}^{\mathrm{m}}$ - fração de volume da matéria orgânica, 0,03

$\theta^{\circ} \quad$ umidade volumétrica do solo, $\mathrm{m}^{3} \mathrm{~m}^{-3}$

\section{Resultados E DiscussÃo}

Na Figura 2 é apresentada a evolução da precipitação pluvial e da umidade volumétrica do solo durante o período de 6/7/2005 a 24/11/2005.

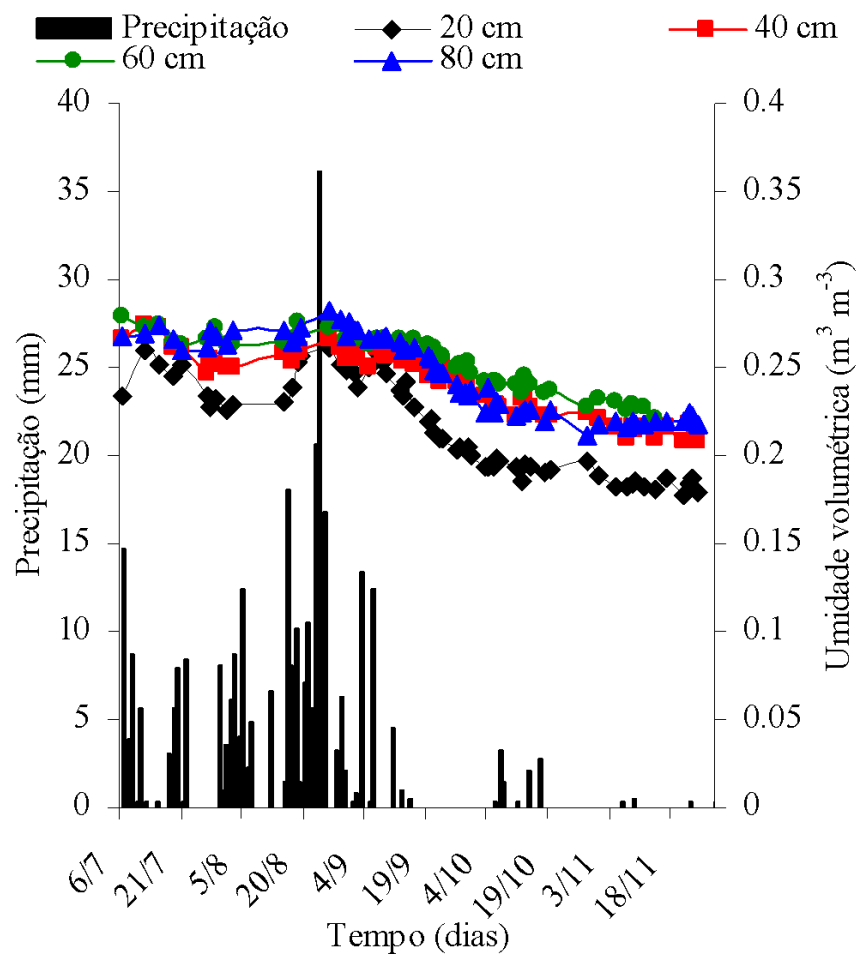

Figura 2. Evolução da precipitação pluvial e da umidade volumétrica do solo nas profundidades de 0,20; 0,40; 0,60 e 0,80 m, no período de 06/07/2005 a 24/11/2005, em Areia, PB

Observa-se, nesta figura, que o período de maior pluviosidade aconteceu entre os dias 15/08/2005 a 25/08/2005 com um total, neste período, de $135,9 \mathrm{~mm}$. Durante o estudo verificou-se que dos 141 dias, 52 foram com chuvas e 10 dias tiveram precipitações acima de $10 \mathrm{~mm}$ e 42 dias com precipitação de até $10 \mathrm{~mm}$. A quantidade total de precipitação pluvial foi de $308,4 \mathrm{~mm}$ sendo que o maior evento pluviométrico (36,1 mm) ocorreu no dia 24/08/2005. A ocorrência de longos períodos chuvosos é uma característica de região de brejos porém as chuvas são, em geral, de baixa intensidade (Santos et al., 2006).

Quanto à umidade volumétrica do solo constata-se que a profundidade de $0,20 \mathrm{~m}$ foi a que apresentou maior variação em resposta ao fornecimento de água via precipitação pluvial, e provável retirada, via evaporação direta e transpiração das plantas de mamona.

$\mathrm{O}$ processo de secagem da água do solo iniciouse de forma contínua logo após o dia 5/9/2005, quando as chuvas praticamente cessaram. A maior variação da umidade volumétrica na profundidade de $0,20 \mathrm{~m}$ se deve, provavelmente, ao fato de que a atmosfera apresentou, neste período (5/9/2005 a 23/11/2005) um grande déficit de água, ao passo que no solo a água nem sempre está disponível para suprir tal demanda atmosférica porque, sempre que o solo seca o suprimento de água na superfície do solo se vai 
restringindo e a condutividade hidráulica começa a influenciar a evaporação. Isto se justifica, segundo Idso et al. (1974), porque a secagem da camada superficial ocorre em três fases distintas, em que a primeira fase corresponde à evaporação potencial, a partir de um solo úmido, e é controlada pelas condições climáticas; a segunda fase se refere a um estado mais seco da superfície e a taxa de evaporação é, em parte, controlada pelo solo e na terceira fase a taxa de evaporação é controlada pelos mecanismos de transferência de vapor e de adsorção no seio da matriz sólida no solo, sendo que esta fase diz respeito a um estado muito seco da superfície do solo; já em relação às profundidades de 0,40; 0,60 e 0,80 m, a variação da umidade volumétrica é idêntica nessas três camadas. Este comportamento é devido, sem dúvida, à barreira física proporcionada pelas profundidades adjacentes reduzindo a condutividade hidráulica nessas profundidades, tal como pela textura, já que nessas profundidades há um teor maior de argila (Tabela 1) promovendo, desta maneira, maior retenção e armazenamento de água no solo, por mais tempo.

Os componentes do balanço hídrico em solo cultivado com mamona durante o período de estudo, são apresentados na Tabela 3 .

Tabela 3. Componentes do balanço hídrico em solo cultivado com mamona durante o período de 06/07/2005 a 24/11/2005 em Areia, PB

\begin{tabular}{crrrr}
\hline Subperíodos & \multicolumn{1}{c}{$\mathbf{P}$} & \multicolumn{1}{c}{$\boldsymbol{\Delta} \mathbf{A}$} & $\mathbf{D} / \mathbf{A C}$ & \multicolumn{1}{c}{$\mathbf{E T}$} \\
1 & 33,53 & 2,46 & $-0,07$ & 30,99 \\
2 & 25,15 & 2,56 & $-0,04$ & 22,55 \\
3 & 18,80 & $-12,82$ & $-0,04$ & 31,57 \\
4 & 40,39 & 3,13 & $-0,01$ & 37,25 \\
5 & 134,36 & 14,03 & $-0,02$ & 120,32 \\
6 & 26,42 & $-8,31$ & $-0,02$ & 34,71 \\
7 & 18,54 & $-10,58$ & $-0,01$ & 29,11 \\
8 & 0,00 & $-8,53$ & $-0,01$ & 8,53 \\
9 & 0,25 & $-4,43$ & 0,01 & 4,70 \\
10 & 9,91 & $-2,02$ & 0,05 & 11,98 \\
11 & 0,00 & $-1,09$ & 0,02 & 1,11 \\
12 & 0,25 & $-5,14$ & 0,04 & 5,44 \\
13 & 0,51 & $-1,27$ & 0,09 & 1,87 \\
14 & 0,25 & $-2,05$ & 0,09 & 2,39 \\
Total & 308,35 & $-34,06$ & $-0,09$ & 342,52 \\
\hline
\end{tabular}

$\mathrm{P}=$ precipitação; $\Delta \mathrm{A}=$ variação do armazenamento de água no solo; $\mathrm{D}=$ drenagem (valores negativos); $A C$ = ascensão capilar (valores positivos); $E T$ = evapotranspiração

Observa-se que os maiores eventos de precipitação pluvial ocorreram entre o $1^{\circ}$ e o $7^{\circ}$ subperíodos sendo que os maiores eventos pluviométricos foram nos subperíodos 4 e 5, com 40,4 e 134,4 mm, respectivamente. Esses valores de precipitação estão abaixo dos sugeridos por Távora (1982) ao comentar que uma precipitação de 400 a $500 \mathrm{~mm}$ até a fase de floração é indicada para a mamona produzir com viabilidade econômica; no entanto, a precipitação ocorrida até esta fase foi de apenas 296,00 mm.

A variação de armazenamento de água no solo $(\Delta \mathrm{A})$ apresentou valores positivos nos subperíodos 1, 2, 4 e 5, com 2,$46 ; 2,56 ; 3,13$ e 14,03 mm, respectivamente. Os subperíodos $3,6,7,8,9,10,11,12,13$ e 14, apresentaram valores negativos de armazenamento, com -12,82;-8,31; -10,58; -8,53; -4,43; $-2,02 ;-1,09 ;-5,14 ;-1,27$ e $-2,05 \mathrm{~mm}$, respectivamente. As variações do armazenamento de água no solo seguiram as variações da precipitação pluvial.
A evapotranspiração da cultura (ET) teve seus maiores valores nos mesmos subperíodos em que ocorreram as maiores precipitações. Esses maiores valores de evapotranspiração em virtude das maiores precipitações pluviais ocorrem devido à maior evaporação nas camadas superficiais até os $40 \mathrm{~cm}$ de profundidade (Cruz et al., 2005a). Observa-se ainda, nos subperíodos 12, 13 e 14, que os valores de evapotranspiração são muito baixos uma vez que nesses subperíodos não ocorreram precipitações sendo que a água necessária para esta evapotranspiração provavelmente veio das camadas mais profundas $(0,60$ e $0,80 \mathrm{~cm})$ as quais apresentam maior capacidade de retenção contribuindo para a absorção de água pelo sistema radicular da cultura.

A ET total no período de 06/07/2005 a 24/11/2005 foi de $342,52 \mathrm{~mm}$ com valor médio de $2,43 \mathrm{~mm} \mathrm{~d}^{-1}$. Oliveira (2007) trabalhando na mesma área experimental e com a mesma variedade de mamona, utilizou o método do balanço de energia razão de Bowen e encontrou valor total de ET de $315,4 \mathrm{~mm}$ e médio de $2,24 \mathrm{~mm} \mathrm{~d}^{-1}$, ou seja, valores muito próximos dos desta pesquisa.

Os valores de fluxo de água na base do solo seguiram a distribuição da precipitação pluvial uma vez que nos subperíodos 1 a 8 ocorreram valores negativos (total no período $-0,21 \mathrm{~mm}$ ), ou seja, drenagem (D) e nos subperíodos 9 a 14 ocorreram valores positivos (total no período $0,30 \mathrm{~mm}$ ), indicando ascensão capilar (AC). No entanto, esses valores foram mínimos e em relação ao termo drenagem eram previstos maiores valores para este tipo de solo, principalmente no subperíodo 5, uma vez que neste subperíodo ocorreu o maior valor total de precipitação pluvial $(134,4 \mathrm{~mm}$ ou 43,26\% do total precipitado) e por esta classe de solo apresentar alta capacidade de infiltração e ser bem drenado naturalmente.

Os baixos valores de drenagem encontrados nesta pesquisa podem ser devidos a vários fatores (umidade volumétrica do solo, potencial matricial, condutividade hidráulica, etc.) que, juntos ou isoladamente, contribuíram para a pobre estimativa deste termo (Reichardt et al., 1979; Netto et al., 2000).

Segundo Reichardt et al. (1979) a maior dificuldade na elaboração de balanços de água no solo se encontra na estimativa do termo drenagem obtido a partir da equação de Darcy, em razão dos erros cometidos na estimativa do gradiente de potencial e principalmente na escolha do valor da condutividade hidráulica.

Dados referentes à ET média diária da mamona e da evapotranspiração de referência (ETo) para as diferentes fases fenológicas são apresentados na Figura 3. Observa-se que a ET média na fase vegetativa foi $4,8 \mathrm{~mm} \mathrm{~d}^{-1}$, sendo que esta fase teve 54 dias de duração; já na fase de floração, que teve 14 dias de duração, a ET média foi $2,8 \mathrm{~mm} \mathrm{~d}^{-1}$. Na fase de enchimento das bagas a ET foi $0,6 \mathrm{~mm} \mathrm{~d}^{-1}$, tendo esta fase 49 dias enquanto na fase de maturidade fisiológica a ET foi 0,4 $\mathrm{mm} \mathrm{d}^{-1}$, a qual teve um período de 24 dias.

Em relação aos valores de ETo os mesmos foram bem superiores aos de ET nas fases de enchimento das bagas e de maturidade fisiológica indicando que nessas fases a mamona passou por estresse hídrico. O valor total de ETo em todo o ciclo da mamona foi de 455,4 $\mathrm{mm}$, com valor médio de 3,3 $\mathrm{mm} \mathrm{d}^{-1}$. 


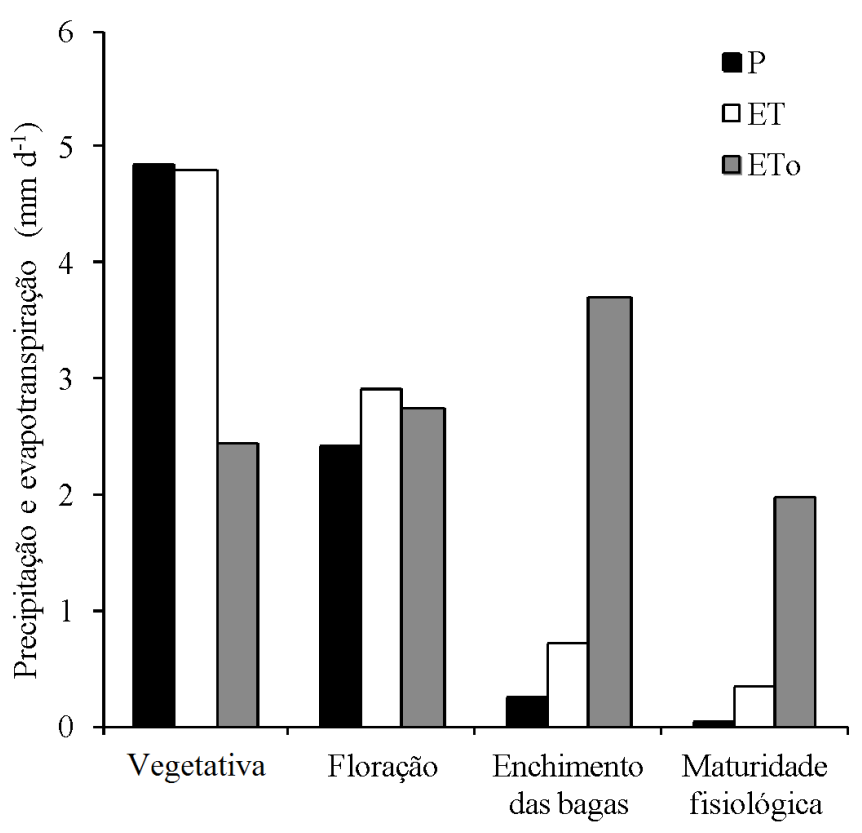

Fases Fenológicas

Figura 3. Média diária da precipitação pluvial (P), evapotranspiração real (ET) e de referência (ETo) para as diferentes fases fenológicas da cultura da mamona em Areia, PB

Os maiores valores de ET na fase vegetativa podem ser explicados pelo fato da mamoneira, no início do ciclo vegetativo, ser exigente em água (Távora, 1982) para que seu desenvolvimento vegetativo seja o máximo possível ocasionando, portanto, um aumento na evapotranspiração nesta fase. Além disso, nesta fase ocorreram maiores valores de precipitação pluvial o que, como já referido, causou aumento na ET. Já o decréscimo da evapotranspiração no final do ciclo ocorre não somente devido às baixas precipitações pluviais mas também à senescência das folhas, reduzindo a área foliar e, consequentemente, a área exposta à transpiração (Lima et al., 2006b).

\section{Conclusões}

1. A evapotranspiração da mamona seguiu as variações da precipitação pluvial e foi, em média, de $2,43 \mathrm{~mm} \mathrm{~d}^{-1}$, com valor total de $342,5 \mathrm{~mm}$.

2. A redução da evapotranspiração da mamona na fase de desenvolvimento reprodutivo coincidiu com o período de maior déficit hídrico do solo.

3. Em relação aos outros componentes do balanço hídrico verificou-se que as variações do armazenamento de água no perfil do solo seguiram as variações da precipitação pluvial e que o fluxo de água na base do solo (ascensão capilar ou drenagem) teve valores muito pequenos.

\section{Agradecimentos}

À Coordenação de Aperfeiçoamento de Pessoal de Nível Superior - CAPES, pela concessão de bolsa de Mestrado ao primeiro autor; à Fundação de Apoio à Pesquisa do Estado da Paraíba - FAPESQ (Termo de concessão 021/05, Edital 002/03 - FAPESQ/MCT/CNPq) e ao Conselho Nacional de Desenvolvimento Científico e Tecnológico - CNPq (Processos $\mathrm{n}^{\text {os }} 556293 / 2006-1,486128 / 2006-7$ e $151494 / 2007-0$ ), pela concessão de recursos financeiros e de bolsas aos terceiro e quarto autores e ao Programa de Pós-Graduação em Manejo de Solo e Água do Centro de Ciências Agrárias da UFPB, pela estrutura didático-científica.

\section{LITERATURA CITADA}

Allen, R. G.; Pereira, L. S.; Raes, D.; Smith, M. Crop evapotranspiration: Guidelines for computing crop water requirements. Rome: FAO, 1998. 300p. FAO. Irrigation and Drainage Paper, 56.

Azevedo, D. M. P.; Lima, E. F. O Agronegócio da Mamona no Brasil. Brasília: Embrapa Informação Tecnológica, 2001. 350p.

Barros Júnior, G.; Guerra, H. O. C.; Cavalcanti, M. L. F.; Lacerda, R. D. de. Consumo de água e eficiência do uso para duas cultivares de mamona submetidas a estresse hídrico. Revista Brasileira de Engenharia Agrícola e Ambiental v.12, p.350-355, 2008.

Beltrão, N. E. de M.; Souza, J. G.; Santos, J. W.; Jerônimo, J. F.; Costa, F. X.; Lucena, A. M. A.; Queiroz, U. C. Fisiologia da mamoneira, cultivar BRS 149 Nordestina, na fase inicial de crescimento, submetida a estresse hídrico. Revista Brasileira de Oleaginosas e Fibrosas, v.7, p.659-664, 2003.

Brito, A. S.; Libardi, P. L.; Ghiberto, P. J. Componentes do balanço de água no solo com cana-de-açúcar, com e sem adubação nitrogenada. Revista Brasileira de Ciência do Solo, v.33, p.295-303, 2009.

Burdine, N. T. Relative permeability calculation from size distribution data. América Institute Mining and Metallurgy Engineering, v.198, p.71-78. 1953.

Cintra, F. L. D.; Libardi, P. L.; Saad, A. M. Balanço hídrico no solo para porta-enxertos de citros em ecossistema de tabuleiro costeiro. Revista Brasileira de Engenharia Agrícola e Ambiental, v.4, p.23-28, 2000.

Cruz, A. C. R.; Libardi, P. L.; Carvalho, L. A.; Rocha, G. C. Balanço de água no volume de solo explorado pelo sistema radicular de uma planta de citros. Revista Brasileira de Ciência do Solo, v.29, p.1-10, 2005a.

Cruz, A. C. R.; Libardi, P. L.; Rocha, G. C.; Carvalho, L. A. Evapotranspiração real de uma cultura de laranja em produção num Latossolo Vermelho-Amarelo. Revista Brasileira de Ciência do Solo, v.29, p.659-668, 2005 b.

EMBRAPA - Empresa Brasileira de Pesquisa Agropecuária. Centro Nacional de Pesquisa de Solos. Manual de métodos de análise do solo. 1.ed. Rio de Janeiro: EMBRAPA, 1997. $212 p$.

EMBRAPA - Empresa Brasileira de Pesquisa Agropecuária. Centro Nacional de Pesquisa de solos. Sistema brasileiro de classificação de solos. 2.ed. Rio de Janeiro: EMBRAPA, 2006. 306p.

Genuchten, M. Th. van. A closed-form equation for predicting the hydraulic conductivity of unsaturated soils. Soil Science Society of America Journal, v.44, p.892-898, 1980 . 
Haverkamp, R.; Ross; P. J.; Smettem, K. R. J.; Parlange, J. Y. Three dimensional analysis of infiltration from the disc infiltrometer. 2. Physically based infiltration equation. Water Resources Research, v.30, p.2931-2935, 1994.

Idso, S. B.; Reginato, R. J.; Kimball, B. A.; Nakayama, F.S. The three stages of drying of field soil. Soil Science Society of America Proceedings, v.38, p.831-835, 1974.

IMSL - International Mathematics and Statistics Library. User manual. Houston: IMSL, 1989. 233p.

Krishnan, P.; Meyers, T. P.; Scott, R. L.; Kennedy, L.; Heuer, M. Energy exchange and evapotranspiration over two temperate semi-arid grasslands in North America. Agricultural and Forest Meteorology, v.153, p.31-44, 2012.

Lima, J. R. S.; Antonino, A. C. D.; Andrade A. P.; Souza, C.; Soares, W. A.; Souza, E. S.; Silva, I. F. Comparação da sonda de nêutrons e de sensores tipo TDR para a determinação dos componentes do balanço hídrico no solo e evapotranspiração do feijão caupi. Agropecuária Técnica, v.27, p.21-29, 2006a.

Lima, J. R. S.; Antonino, A. C. D.; Lira, C. A. B. O.; Souza, E. S.; Silva, I. F. Balanço de energia e evapotranspiração de feijão caupi sob condições de sequeiro. Revista Ciência Agronômica, v.42, p.65-74, 2011.

Lima, J. R. S.; Antonino, A. C. D.; Soares, W. A.; Souza, E. S.; Lira, C. A. B. O. Balanço hídrico no solo cultivado com feijão caupi. Revista Brasileira de Ciências Agrárias, v.1, p.89-95, 2006b.

Moroke, T. S.; Schwartz, R. C.; Brown, K. W.; Juo, A. S. R. Water use efficiency of dryland cowpea, sorghum and sunflower under reduced tillage. Soil \& Tillage Research, v.112, p.76-84, 2011.

Netto, A. M.; Antonino, A. C. D.; Audry, P.; Carneiro, C. J. G.; Dall'olio, A. Condutividade hidráulica não saturada de um Podzólico Amarelo da zona da mata norte de Pernambuco. Pesquisa Agropecuária Brasileira, v.35, p. 1221-1228, 2000.
Oliveira, I. A. de. Balanço de energia em mamona, cultivada sob condições de sequeiro, no Brejo Paraibano. Areia: UFPB, 2007. 56p. Dissertação Mestrado

Oliveira, I. A.; Lima, J. R. S.; Silva, I. F.; Antonino, A. C. D.; Gouveia Neto, G. C.; Lira, C. A. B. O. Balanço de energia em mamona cultivada em condições de sequeiro no Brejo Paraibano. Revista Brasileira de Ciências Agrárias, v.4, p.185-191, 2009.

Oresegun, M. O. Radiation safety of soil moisture neutron probes. In: International Atomic Energy Agency. Comparison of soil water measurement using the neutron scattering, time domain reflectometry and capacitance methods. Vienna: IAEA, 2000. Cap.8, p.139-146.

Reichardt, K.; Libardi, P. L.; Saunders, L. C. U.; Cadimaz, A. Dinâmica da água em solo cultivado com milho. Revista Brasileira de Ciência do Solo, v.3, p.1-5, 1979.

Santos, A. C.; Andrade, A. P.; Silva, I. F.; Azeredo, G. A. Variabilidade temporal da precipitação pluviométrica e rendimento do algodoeiro herbáceo em diferentes sistemas de manejo de solo e de adubação nitrogenada. Pesquisa Agropecuária Tropical, v.36, p.123-129, 2006.

Távora, F. J. A. A cultura da mamona. Fortaleza: EPACE, 1982. 111p.

Vries, D. A. de. Thermal properties of soils. In: Wijk, W. R. van. Physics of Plant Environment. Amsterdam: NorthHolland, 1966. p.210-233.

Ward, P. R.; Flower, K. C.; Cordingley, N.; Weeks, C.; Micin S. F. Soil water balance with cover crops and conservation agriculture in a Mediterranean climate. Field Crops Research, v.132, p.33-39, 2012.

Wessolek, G.; Plagge, R.; Leij, F. J.; Genuchten, M. Th. van. Analyzing problems in describing field and laboratory measured soil hydraulic properties. Geoderma, v.64, p.93$110,1994$. 